\title{
Effect of stool size and consistency on defecation
}

\author{
J J BANNISTER, P DAVISON, J M TIMMS, C GIBBONS, AND N W READ \\ From the Departments of Surgery and Physiology, Royal Hallamshire Hospital, Sheffield
}

SUMmaRY The ability of subjects to expel from the rectum objects simulating stools of different characteristics was assessed in paired studies carried out in a total of 58 normal subjects and 25 young women with severe constipation. Our results showed that a lower percentage of normal subjects and a lower percentage of constipated patients were able to pass a $1.8 \mathrm{~cm}$ incompressible sphere compared with a $50 \mathrm{ml}$ deformable balloon, although constipated patients found it more difficult than normal subjects to expel both types of simulated stool. It was also more difficult for normal subjects to pass a soft compressible silicon rubber simulated stool than a stool made up of a similar volume of incompressible $1 \mathrm{~cm}$ wooden spheres contained in a cylindrical latex envelope, but both objects were much easier to pass than the same number of $1 \mathrm{~cm}$ spheres placed loose within the rectum. When normal subjects were instructed to expel single incompressible spheres of different sizes placed in the rectal ampulla, the intrarectal pressure and the time needed to pass these objects varied inversely with their diameter. These results suggest that more effort is required to expel stools from the rectum if they are small and hard than if they are large and soft.

Constipated patients or patients with the irritable bowel syndrome (IBS) often complain of difficulties with defecation. In recent studies, $38 \%$ of patients with IBS' and $80 \%$ of women with severe idiopathic constipation' said they had to strain for long periods of time in order to defecate. These difficulties may be related at least in part to the nature of the stool passed. Over $80 \%$ of patients with constipation' ${ }^{2}$ pass separate hard pellets from the rectum. Proctoscopy often shows the presence of such pellets in the rectum, suggesting that they are formed in the sigmoid and rectum and are not moulded to any large extent during passage through the anus. To investigate whether the nature of the stool has any effect on defecation we devised a series of studies, in which we measured the ability of normal subjects and constipated patients to expel from the rectum objects simulating stools of differing size and consistency. Similar studies were carried out in a group of young constipated patients to investigate whether these patients had any greater difficulty in expelling the same objects than normal subjects.

Address for correspondence: Dr N W Read. Sub-department of Human Gastrointestinal Physiology \& Nutrition. Floor K. Royal Hallamshire Hospital. Shefficld $S 10$ () $2 \mathrm{JF}$.

Received for publication 19 February 1987.

\section{Methods}

SUBJECTS

The normal subjects consisted of 58 healthy volunteers ( 28 men, 30 women, aged $20-90$ years) who had no history of difficulties with defecation, and who did not suffer from gastrointestinal problems.

The constipated patients were 25 women (aged 15-54 years) who complained of severe constipation refractory to treatment with dietary fibre (at least 30 $\mathrm{g} /$ day) and laxatives.

EXPULSION OF CYLINDRICAL WATER FILLED

BALLOONS AND A PLASTIC SPHERE

Studies were carried out in 36 normal subjects ( 25 women, 11 men) and 25 constipated patients. Cylindrical balloons containing 50 and $25 \mathrm{ml}$ water simulated soft deformable stools of different sizes. The larger balloon was constructed from $10 \mathrm{~cm}$ of unstretched latex condom and the smaller from an unstretched rubber finger cot. Each balloon was tied around a polythethylene tube (od $2 \mathrm{~mm}$ ). The deflated balloon and its attached catheter was inserted digitally into the low rectum using copious amounts of lubrication (KY Jelly, Johnson and Johnson), and was then filled with $50 \mathrm{ml}$ or $25 \mathrm{ml}$ 
warm $\left(37^{\circ} \mathrm{C}\right)$ water. A hard polyethylene sphere 1.8 $\mathrm{cm}$ in diameter simulated the hard pellets that many constipated patients pass. Each object was inserted into the rectum on separate occasions and in random order, and was pulled down gently so that it lay in the rectal ampulla. The subject was then allowed to sit up on a commode and left alone in a cubicle, although the technician remained within hailing distance in the adjacent room. When the subject felt at ease he/she started a stop clock and attempted to expel the object from the rectum. The clock was stopped as soon as the simulated stool was passed. If the object was not expelled within 5 minutes, it was removed by traction on the manometric tubing or chain. The procedure was then repeated with the other simulated stools.

EXPULSION OF SIMULATED STOOLS OF THE SAME VOLUME, BUT DIFFERENT COMPOSITION AND CONSISTENCY

Studies were carried out in nine male normal volunteers. Having evacuated the bowel before beginning the experiment, the subject lay in the left lateral position. A narrow manometric catheter (od $2 \mathrm{~mm}$ ) with a distal side opening port was inserted into the rectum and perfused with distilled water at a rate of $0.2 \mathrm{ml} / \mathrm{min}$ using a low compliance hydraulic capillary perfusion system (Andorfer Medical Specialities Inc., Greendale, Wisconsin, USA). A pressure transducer (Druck Ltd, Groby, Leics, UK) situated in the perfusion line measured rectal pressure and the signal was amplified and displayed on a chart recorder (Lectromed MT8 PX, Ormed Ltd, Welwyn Garden City, UK).

A soft, deformable, cylindrical object made of silicon rubber tapered at either end (volume $30 \mathrm{ml}$ by immersion) simulated a normal stool. A cord attached to one end facilitated its removal from the rectum. Thirty wooden beads, each $1 \mathrm{~cm}$ in diameter contained inside a double layered envelope constructed from two unstretched rubber finger cots simulated a hard stool. The individual components of this object were incompressible but the object could be deformed by moving the beads within the finger cots. A cord was attached to a bead inside the finger cots to enable this simulated stool to be withdrawn from the rectum. The packed bead volume (by immersion) was the same as the silicon stool and shape of the simulated hard stool was adjusted to match the shape of the simulated normal stool. The third and final simulation consisted of 30 loose $1 \mathrm{~cm}$ beads. Each simulation was inserted into the rectum using generous amounts of lubrication (KY Jelly, Johnson and Johnson) and where appropriate pulled down gently so that it lay in the rectal ampulla.

The subject then sat on a commode in a private cubicle, although the technician was within hailing distance in an adjacent room. In the first part of the study, the subject was allowed to strain normally to expel the objects from the rectum. In the case of the loose beads, he was told to strain until he no longer had a desire to defecate, and the percentage of beads recovered was recorded. The subject recorded the total time spent straining on a hand held stop watch. Each simulation was inserted into the rectum on at least two occasions, and the order of insertions was randomised. The shortest time taken to pass each object was recorded.

The experiment was repeated in order to measure the minimum intrarectal pressure that was required to pass the simulated stools. The subject was trained to increase his intra-abdominal pressure in $20 \mathrm{mmHg}$ stages as indicated by lines drawn on the rectal pressure trace. The subject maintained the rectal pressure at each prescribed level for a total of 30 seconds, and if he had not evacuated the object within that time, he then increased the pressure by 20 $\mathrm{mmHg}$ and attempted to pass the object again. The whole procedure was repeated at increasing intraabdominal pressures until the object was evacuated. If the subject strained above the prescribed limits, the results were ignored and the test was repeated.

EXPULSION OF SPHERES OF DIFFERENT SIZES Studies were carried out in eight male and five female normal volunteers. With the subject lying in the left lateral position, a narrow manometric catheter (od $2 \mathrm{~mm}$ ), inserted into the rectum and pressures were recorded as described above.

Hard polyethylene spheres, $0 \cdot 5,1 \cdot 0,1 \cdot 5,1 \cdot 8$, and $2.5 \mathrm{~cm}$ in diameter, each with a nylon cord attached for ease of removal, were used to simulate hard stool pellets of different sizes. Each sphere was placed in the rectum on separate occasions in random order and using generous amounts of lubrication. The subject then sat on a commode and raised the intraabdominal pressure stepwise by blowing into the tubing attached to a sphygmomanometer and raising the column of mercury in increments of $20 \mathrm{mmHg}$, taking care to maintain the pressure using the abdominal muscles and the diaphragm and not by compressing the tongue against the palate. These increases in intra-abdominal pressure were recorded by the intrarectal pressure sensor and there was a highly significant correlation between the pressure in the manometer and the pressure recorded in the rectum $(r=0.85, p<0.001)$. The subject was not allowed to strain and raise the intra-abdominal pressure above the prescribed limit and was allowed three minutes to expel the sphere at each level of pressure. The minimum intrarectal pressure required to pass each sphere was recorded. In a separate series of studies, the subjects were permitted to strain 
normally, and the minimum time taken to pass each sphere was recorded.

\section{STATISTICAL ANALYSIS}

Data which could be expressed in a $2 \times 2$ contingency table was analysed using the $\chi^{2}$ test with Yates correction for small numbers. Normally distributed data were analysed using the paired or unpaired (as appropriate) Student's $t$ test. Non-parametric paired data was analysed using the Wilcoxon's signed-ranksum test.

\section{Results \\ EXPUISION OF CYLINDRICAL WATER FILLED BALLOONS VERSUS PLASTIC SPHERES}

\section{Normal patients}

A significantly lower number of normal subjects could expel the $1.8 \mathrm{~cm}$ sphere compared with the 50 $\mathrm{ml}$ balloon from the rectum within five minutes $(p<0 \cdot 001)$ (Fig. 1). There were no significant differences in the number of subjects who could expel the $25 \mathrm{ml}$ balloon compared with either the $50 \mathrm{ml}$ balloon or the $1.8 \mathrm{~cm}$ sphere (Fig. 1). No difference was found in the ability of men compared with women to pass the simulated stools.

\section{Constipated patients}

A significantly lower number of constipated patients could pass the $1.8 \mathrm{~cm}$ sphere than the $50 \mathrm{ml}$ balloon from the rectum within five minutes $(p<0.05)$ (Fig. 1). Again, there were no significant differences in the number of patients who could expel the $25 \mathrm{ml}$ balloon compared with either the larger balloon or the sphere.

When the constipated patients were compared with normal volunteers of similar age and sex (19 women, aged 20-55 years), a significantly lower proportion of patients were able to expel either the 50 $\mathrm{ml}$ balloon $(\mathrm{p}<0.05)$ or the $1.8 \mathrm{~cm}$ sphere $(\mathrm{p}<0.01)$.

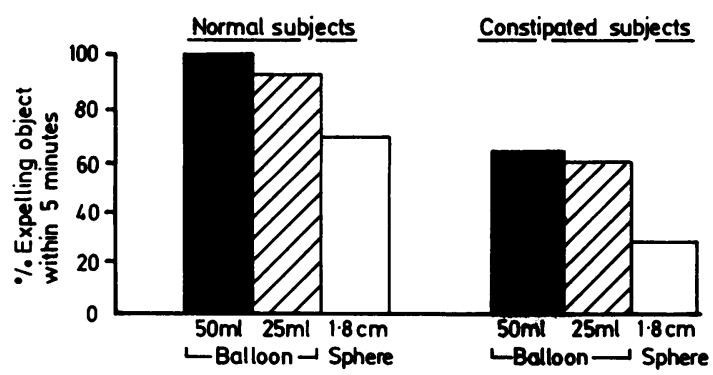

Fig. 1 Percentage of normal (left) and constipated (right) subjects able to expel a $50 \mathrm{ml}$ balloon, a $25 \mathrm{ml}$ balloon or a $1.8 \mathrm{~cm}$ sphere from the rectum within five minutes.
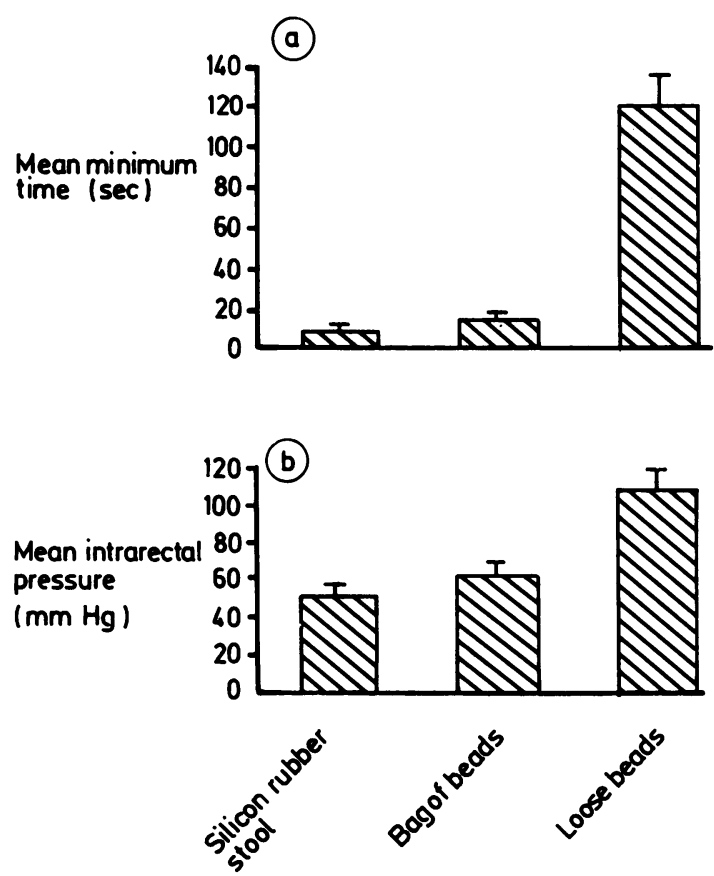

Fig. 2 Mean minimum (a) time (mean $\pm S E M$ ) (upper graph) and (b) intrarectal pressure (mean $\pm S E M$ ) (lower graph) required to expel a $30 \mathrm{ml}$ silicon rubber simulated stool and a similar volume of incompressible $1 \mathrm{~cm}$ beads in a rubber finger cot, in nine normal male subjects. Also shown is the minimum time and intrarectal pressure required for subjects to feel they had expelled all the loose beads from the rectum.

\section{EXPULSION OF STOOL OF DIFFERENT , CONSISTENCY}

All subjects were able to pass the silicon rubber stool and the bag of beads, but only one subject was able to expel all the loose beads. Between 30 and 100\% ( median $=67 \%$ ) of the beads were recovered when the subjects strained normally, and between 27 and $90 \%$ (median $=68 \%$ ) of the beads were recovered when intrarectal pressure was constrained.

When subjects strained normally, they took longer to expel the bag of beads than the silicon stool $(p<0.05)$ (Fig. 2a). These times were both much shorter, however, than the times taken for subjects to evacuate sufficient loose beads to abolish the desire to defecate ( $<<0 \cdot 01)$ (Fig. 2a).

When the intrarectal pressure was constrained, the minimum pressure required to pass the silicon stool or the bag of beads was significantly less than for the loose beads $(p<0.01)$ (Fig. 2b). There was no significant difference in the minimum pressure required to pass the bag of beads compared with the silicon stool (Fig. 2b). 
EXPULSION OF SPHERES OF DIFFERENT

DIAMETERS

Only three of 13 subjects (two men, one woman) managed to pass the smallest (diameter $0.5 \mathrm{~cm}$ ) sphere, whereas 11 of 13 passed the $1.0 \mathrm{~cm}$ sphere $(\mathrm{p}<0.01$ compared with $0.5 \mathrm{~cm}$ sphere $)$ and all 13 passed the $2.5 \mathrm{~cm}$ sphere $(\mathrm{p}<0.001$ compared with the $0.5 \mathrm{~cm}$ sphere). No sex differences were found in any part of this study.

The minimum intrarectal pressures required to expel each sphere fell as the diameter rose (Fig. 3); the pressure required to expel the $1.0 \mathrm{~cm}$ sphere being significantly greater than that needed to expel the $2.5 \mathrm{~cm}$ sphere $(\mathrm{p}<0 \cdot 05)$. Plotting the minimum intrarectal pressure needed to expel each sphere against the inverse of the radius of that sphere for each subject yielded linear correlation coefficients ranging from 0.75 to 0.99 (median 0.82 ) (Fig. 4).

The minimum time needed to pass each sphere also fell as the diameter rose (Fig. 5); the time needed to expel the $1.0 \mathrm{~cm}$ sphere being significantly greater than that needed to expel the $2.5 \mathrm{~cm}$ sphere $(\mathrm{p}<0 \cdot 01)$.

\section{Discussion}

These studies indicate that people find it easier to expel large deformable stools from the rectum than small hard pellets. Although these differences appear to be predominantly because of stool size, subjects also passed a soft deformable silicon rubber stool in a shorter time, than they took to expel a similar volume of hard, incompressible spheres contained within a finger cot. Longer times and higher pressures were required, however, for subjects to evacuate sufficient

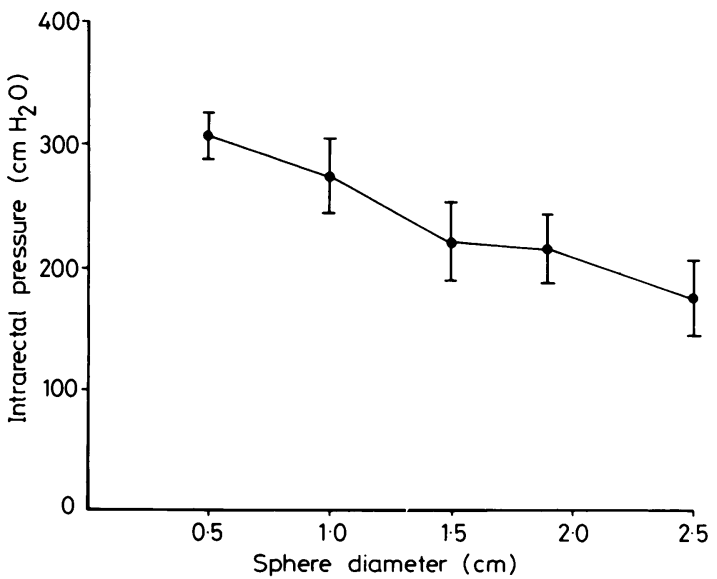

Fig. 3 Minimum intrarectal pressures (mean $\pm S E M$ ) attained by normal subjects whilst passing spheres of varying diameter from the rectum.

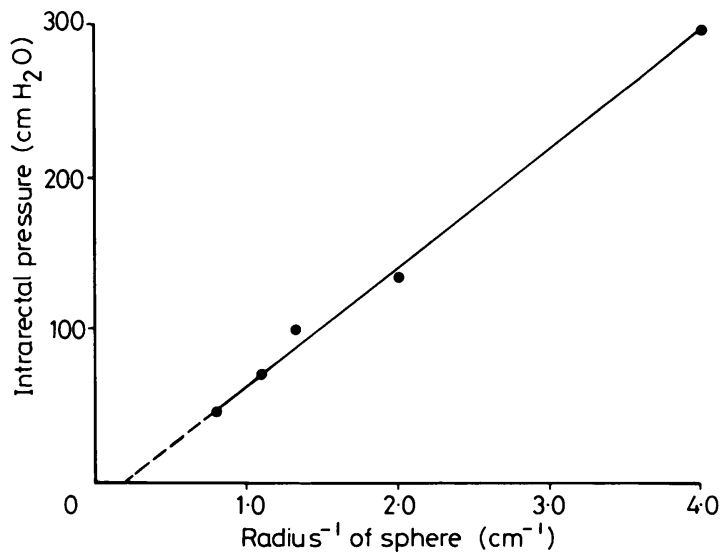

Fig. 4 Minimum intrarectal pressure required to expel spheres of varying diameters from the rectum in one normal subject plotted against the inverse of the radius of the sphere. Linear correlation coefficient $=0.99(p<0 \cdot 001)$.

incompressible spheres lying loose in the rectum to abolish the desire to defecate. This particular observation suggests that the sensation of incomplete evacuation in some but probably not all patients with irritable bowel syndrome may be related to retained faecal pellets.

Studies using spheres of different diameters showed that ease of defecation was directly related to the diameter of the sphere. A sphere of $0.5 \mathrm{~cm}$ in diameter could only be expelled by three of 13

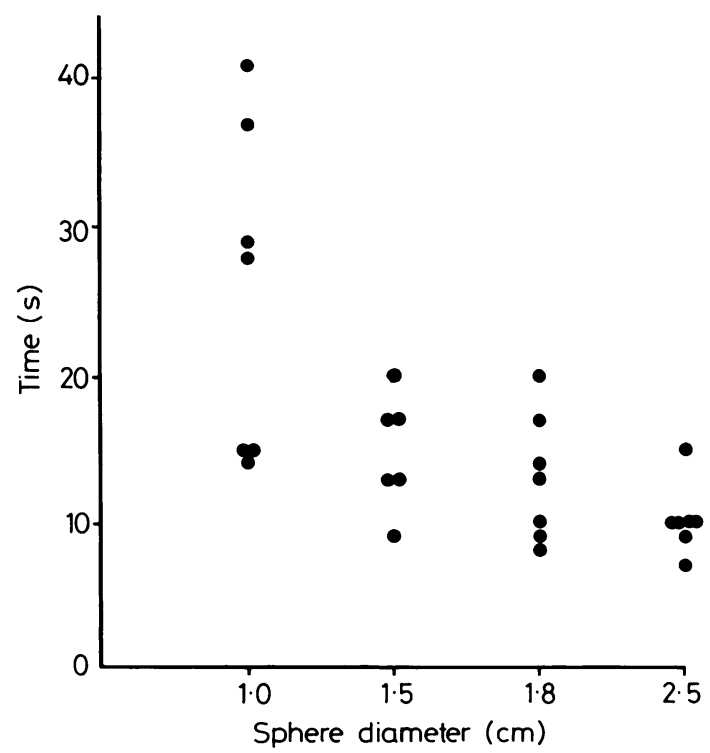

Fig. 5 Minimum times in which seven normal subjects could expel spheres of varying diameter from the rectum. 
normal volunteers: a sphere of $1.0 \mathrm{~cm}$ in diameter could be passed by most volunteers, but larger intraabdominal pressures and longer times were required to pass this object compared with - for example, a $2 \cdot 5$ $\mathrm{cm}$ sphere. The results are supported by theoretical considerations. During defecation, inhibition of the external anal sphincter (EAS) is probably modulated by cortical mechanisms and is thought to be independent of the diameter of the stool. ${ }^{3}$ In fact, the electrical activity of the EAS can be inhibited by 'bearing down' even with no stool in the rectum. ${ }^{+1}$ Inhibition of the internal anal sphincter (IAS), on the other hand, is directly related to the degree of rectal distension, ${ }^{7}$ and is probably mediated by the degree of stimulation of circumferential stretch receptors in the rectal wall. ${ }^{x}$ If this is so, then there should be an inverse relationship between stool circumference (or stool radius) and anal pressure during defecation, and hence the minimum intrarectal pressure required to expel a sphere should also be inversely related to the radius of the stool as observed in our subjects. The same relationship would apply to a cylindrical or sausage shaped stool provided that object approaches the sphincter end on.

The results of this study therefore suggest that the difficulty in defecation experienced by many patients with constipation or IBS may be related in part to the size and consistency of the stool in the rectum; small, hard stools being harder to expel than large soft stools. This would explain how ingestion of a high fibre diet might facilitate defecation in such patients by increasing stool size and making the stool softer and more deformable. ${ }^{9}$ The patients with severe diet and laxative resistant constipation, that we investigated, however, found it more difficult than normal subjects to pass each of the simulated stools. This is presumably related to the observation that many of these patients do not relax their pelvic floor as they attempt to defecate, but obstruct defecation by contracting their external sphincter and puborectalis." "I The force required to propel a solid stool around a sharp anorectal angle and expel it through a contracted sphincter would be extremely high and may be impossible to achieve by normal contraction of abdominal muscles.

\section{References}

1 Cann PA. Thesis: Leeds, 1986.

2 Bannister JJ, Timms JM, Barfield LJ, Donnelly TC, Read NW. Physiological studies in young women with chronic constipation. Int J Colorectal Dis 1986; 1: 175-82.

3 Ihre T. Studies on anal function in continent and incontinent patients. Scand J Gastroenterol 1974; 9: suppl: 25.

4 Floyd WF, Walls EW. Electromyography of the sphincter ani externus in man. J Physiol 1953; 122: 599-609.

5 Parks AG, Porter NH, Melzak J. Experimental study of the reflex mechanism controlling the muscles of the pelvic floor. Dis Colon Rectum 1962; 5: 407-14.

6 Preston DM, Lennard-Jones JE. Anismus in chronic constipation. Dig Dis Sci 1985; 30: 413-8.

7 Meunier P, Marechal JM, De Beaujeu MJ. Rectoanal pressures and rectal sensitivity studies in chronic childhood constipation. Gastroenterology 1979; 77: 330-6.

8 Martelli H, Devroede G, Arhan P, Duguay C, Dornic C, Faverdin C. Some parameters of large bowel motility in normal man. Gastroenterology 1978; 75: 612-8.

9 Davies GJ, Crowder M, Reid B, Dickerson JWT. Bowel function measurements of individuals with different eating patterns. Gut 1986; 27: 164-9.

10 Read NW, Timms JM, Barfield LJ, Donnelly TC, Bannister JJ. Impairment of defecation in young women with severe constipation. (jastroenterology 1986; 90: 53-60. 